\title{
Suggested Gestational Weight Gain for Chinese Women and Comparison with Institute of Medicine Criteria: A Large Population-Based Study
}

\author{
Wei Zheng ${ }^{\text {a Wenyu Huang }}{ }^{\text {a, b }}$ Li Zhang $^{\text {a }}$ Zhihong Tian $^{\mathrm{a}} \quad$ Qi Yan ${ }^{\mathrm{a}}$ \\ Teng Wang $^{\text {a }}$ Guanghui Li $^{\text {a }}$ Weiyuan Zhang ${ }^{\text {a }}$ \\ ${ }^{a}$ Division of Endocrinology and Metabolism, Beijing Obstetrics and Gynecology Hospital, Capital Medical University, \\ Beijing, China; ${ }^{b}$ Division of Endocrinology, Metabolism and Molecular Medicine, Northwestern University Feinberg \\ School of Medicine, Chicago, IL, USA
}

\section{Keywords}

Gestational weight gain · Pregnancy outcome - Large for gestational age $\cdot$ Small for gestation age $\cdot$ Institute of Medicine

\section{Abstract}

Objective: To establish suggested gestational weight gain (GWG) using several distinct methods in a Chinese population. Methods: This study analyzed data from the medical records of singleton pregnancy women during 2011-2017 in Beijing, China. Suggested GWG was calculated using four distinct methods. In method 1, suggested GWG was identified by the interquartile method. Subsequently, risk models for small for gestational age (SGA) and large for gestational age (LGA) with respect to GWG were constructed. GWG was treated as a continuous variable in method 2 , and as a categorized variable in methods 3 and 4. Results: An average GWG of $15.78 \mathrm{~kg}$ with a prevalence of LGA at $19.34 \%$ and SGA at $2.12 \%$ was observed among the 34,470 participants. Methods 1 and 2 did not yield clinically applicable results. The suggested GWGs were 11-17/11-16 kg, 9-19/9-15 kg, 4-12/4-10 kg, and 0-12/0-6 kg by method 3/method 4 for underweight, normal-weight, overweight, and obese wom-

karger@karger.com www.karger.com/ofa

Karger"

BOPEN ACCESS
(C) 2021 The Author(s)

Published by S. Karger AG, Basel

This article is licensed under the Creative Commons AttributionNonCommercial-NoDerivatives 4.0 International License (CC BYNC-ND) (http://www.karger.com/Services/OpenAccessLicense). Usage and distribution for commercial purposes as well as any distribution of modified material requires written permission. en, respectively. The GWG range suggested by method 3 resulted in a larger proportion of participants $(62.03 \%)$ within range, while the suggested GWG range by method 4 was associated with a lower risk of LGA compared to that conferred by the Institute of Medicine (IOM) criteria. Conclusion: This study suggests a modest GWG goal compared to IOM recommendations based on a large Chinese cohort.

(c) 2021 The Author(s)

Published by S. Karger AG, Basel

\section{Introduction}

Inappropriate gestational weight gain (GWG) increases both short-term and long-term health risks of mothers and offspring $[1,2]$. Excessive GWG is associated with increased risk of cesarean delivery [3], postpartum weight retention [4], and large for gestational age (LGA) [3], all of which increase the long-term risk of obesity in both mothers and offspring [5]. In contrast, insufficient GWG increases the risk of small for gestational age (SGA) [3]. Nonetheless, there is no global consensus on the recommended GWG [6]. The Institute of Medicine (IOM) guideline provides the most widely used criteria for GWG [7]. However, it was developed based on Caucasian popu- 


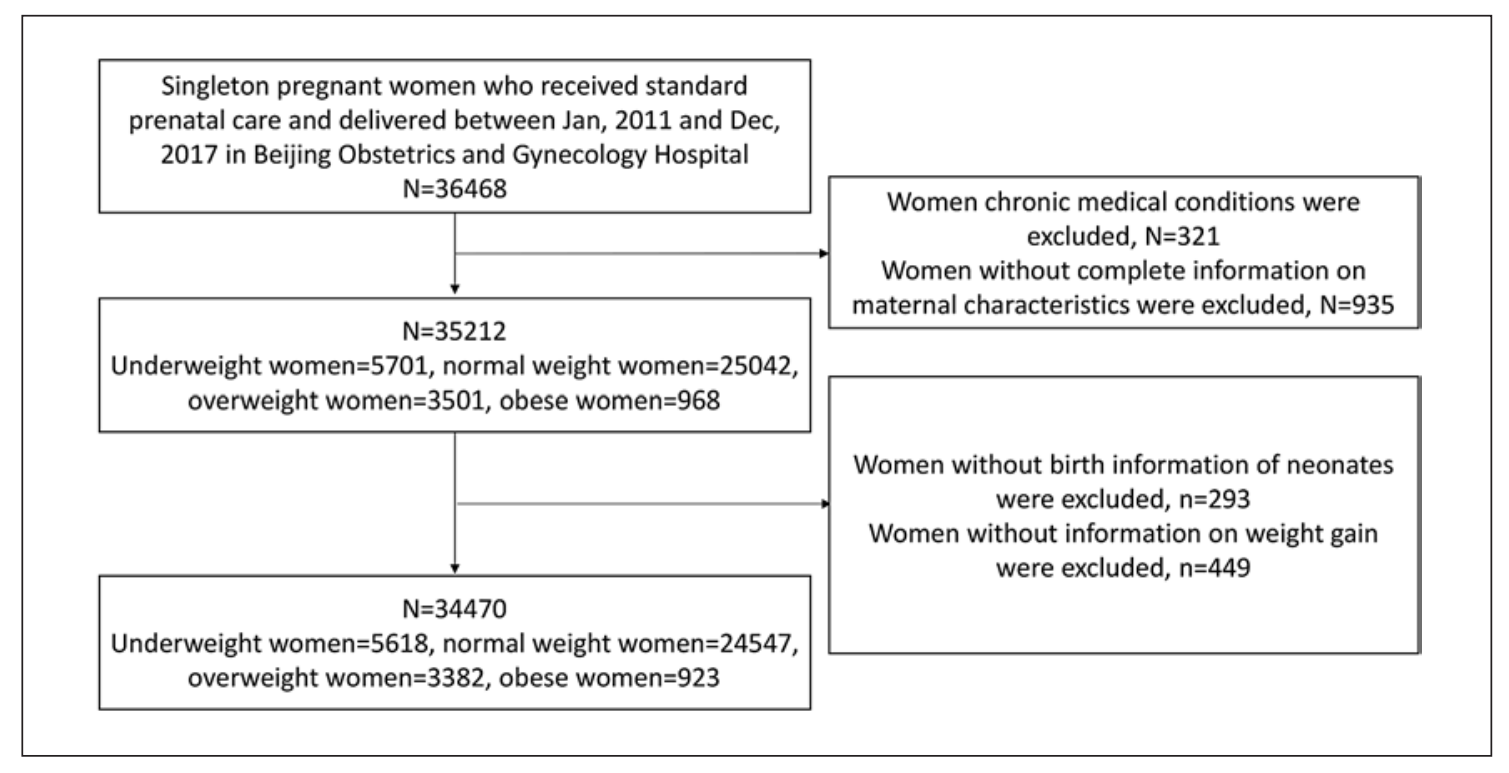

Fig. 1. Flowchart of the participants included in the analysis.

lations and was not intended for use in women who are thinner and shorter than US women [7].

There is a lack of evidence-based guidelines for "optimal" GWG in Asian populations. Recent studies in Japan, Singapore, and Taiwan have indicated that optimal GWG in Asian women may be substantially lower than that recommended by the IOM, especially in overweight and obese women [8-10]. We aimed to establish suggested GWG in a large Chinese population by applying different risk models for GWG-associated perinatal outcomes.

\section{Subjects and Methods}

We used data from singleton pregnancy women having delivered between January 2011 and December 2017 in Beijing Obstetrics and Gynecology Hospital in China. We included women who had received standard prenatal care to ensure they had credible information about prepregnancy weight and predelivery weight. Women with chronic medical conditions including hypertension, type 2 diabetes mellitus, heart disease, or kidney disease were excluded. Women without data regarding baseline characteristics, GWG, or neonatal birthweight were also excluded. The number of participants included is shown in a flowchart (Fig. 1).

Clinical information was collected through medical record review. The collected information included maternal date of birth, height, self-reported prepregnancy weight, predelivery weight, gestational age, and neonatal birth weight in the form of continuous variables, as well as parity, gestational diabetes mellitus (GDM), pregnancy-induced hypertension (PIH), delivery mode (cesarean section or vaginal delivery), and neonatal gender in the form of categorical variables. Prepregnancy body weight was self-reported at the first prenatal visit around the 5th-6th gestational week. Prepregnancy body mass index (PPBMI) was calculated as prepregnancy weight/height squared $\left(\mathrm{kg} / \mathrm{m}^{2}\right)$. The participants were defined as underweight (PPBMI $<18.5 \mathrm{~kg} / \mathrm{m}^{2}$ ), normal weight $\left(18.5 \leq \mathrm{PPBMI}<25 \mathrm{~kg} / \mathrm{m}^{2}\right)$, overweight $\left(25 \leq \mathrm{PPBMI}<30 \mathrm{~kg} / \mathrm{m}^{2}\right)$, or obese (PPBMI $\geq 30 \mathrm{~kg} / \mathrm{m}^{2}$ ). GDM was diagnosed by using the National Diabetes Data Group (NDDG) 2-step criteria [11] and the International Association of Diabetes and Pregnancy Study Groups (IADPSG) criteria in women who delivered before 2012 and since 2012 [12]. PIH was defined as the development of new hypertension after a gestational age of 20 weeks in a previously normotensive woman [13]. Birth weight was classified as low birth weight (LBW; $<2,500 \mathrm{~g}$ ), normal weight $(2,500-4,000 \mathrm{~g})$, and macrosomia $(>4,000 \mathrm{~g})$. LGA and SGA (birthweights greater than or below the 10th percentile for the gestational age) were defined according to an international standard set by Villar et al. [14]. Gestational age $<37$ weeks is defined as preterm.

The data were analyzed using SAS 9.3. Associations between GWG and perinatal outcomes, including LGA, SGA, macrosomia, LBW, preterm delivery, and cesarean section, were evaluated using a logistic regression model adjusted for maternal age, parity, PPBMI, height, GDM, and PIH. Gestational age was also adjusted for when using macrosomia, LBW, and cesarean section as outcomes. GWG within the IOM-recommended range was used as a reference. Subsequently, suggested GWG was calculated using four distinct methods. In method 1, GWG data from subjects with optimal perinatal outcomes (vaginal delivery of a term appropriate-for-gestational-age infant without macrosomia, LBW, GDM, or $\mathrm{PIH}$ ) were used to generate an interquartile (25-75\%) GWG range as the suggested GWG. In method 2, a similar method to the one reported by Beyerlein et al. [15] was used to develop a logistic regression model in order to assess the relationship between GWG and the risk of SGA and LGA, with GWG being a continuous variable which was adjusted for maternal age, parity, PPBMI, height, GDM, and PIH. Suggested GWG is defined by a joint predicted
Zheng et al. 
risk of $20 \%$ for SGA and LGA. In method 3, a logistic regression model was used to evaluate the association of the risk of SGA and LGA with categorized GWG intervals, adjusted for the covariates mentioned for method 2. GWG was categorized in 1- and 2-kg intervals into underweight/normal-weight women and overweight/ obese women, due to the limited sample sizes of the latter groups. IOM-recommended lower and upper limits of GWG for each weight group were set as the reference levels to calculate adjusted odd ratios (ORs) for LGA and SGA, respectively, in each categorized GWG interval. Accordingly, in the determination of adjusted ORs for LGA, $12.0-12.9 \mathrm{~kg}, 11.0-11.9 \mathrm{~kg}, 6.0-7.9 \mathrm{~kg}$, and $4.0-5.9$ $\mathrm{kg}$ were the reference GWG ranges for the underweight, normalweight, overweight, and obese groups, respectively, whereas the ORs for SGA were calculated using 17.0-17.9 kg, $15.0-15.9 \mathrm{~kg}$, $10.0-11.9 \mathrm{~kg}$, and $8-9.9 \mathrm{~kg}$ as the reference ranges for the respective PPBMI groups. Suggested GWG was determined using an OR of 2.0 or above as the cut-off for risk of LGA/SGA compared to the corresponding reference in the group. Method 4 was similar to method 3, except that an OR cut-off of 1.5 for LGA, instead of 2.0, was used to calculate the upper/lower range of suggested GWG. This difference in OR cut-off value between method 3 and method 4 was made due to the high prevalence of LGA in our cohort. After having established the suggested GWG ranges using the aforementioned methods, we calculated the percentage of participants in each PPBMI group who were within the suggested GWG range as determined by each method. We also compared rates of prevalence of LGA and SGA among women who met the suggested GWG ranges according to the IOM criteria or the proposed GWG ranges by $\chi^{2}$ test.

\section{Results}

A total of 34,470 participants were included (Fig. 1). As shown in Table 1, the average GWG was $15.78 \mathrm{~kg}$, with a high prevalence of LGA (19.34\%) and a low prevalence of SGA $(2.12 \%)$.

The majority of pregnant women in our cohort did not gain weight during gestation as recommended by the IOM guideline (Table 2). To be more specific, only $52.56 \%$ of the underweight, $39.63 \%$ of the normal-weight, $26.82 \%$ of the overweight, and $30.55 \%$ of the obese women achieved GWGs that were within the IOM-recommended ranges. Using the women with IOM-recommended GWG as the reference group, perinatal outcomes were compared between the reference group and those who did not achieve the recommended GWG. Excessive GWG was associated with higher risk of LGA and cesarean section in all weight groups, as well as with higher risk of macrosomia in all weight groups except for obese women, while insufficient GWG was associated with elevated risk of SGA and preterm delivery only for underweight and normal-weight women and elevated risk of LBW for normal-weight women (Table 2).

Suggested GWG in Chinese Women
Table 1. Characteristics of the participants

\begin{tabular}{ll}
\hline Participants & 34,470 \\
Age, years & $30.58 \pm 4.17$ \\
Primipara & $29,040(84.25)$ \\
Height, cm & $162.82 \pm 4.79$ \\
Prepregnancy BMI, kg/m ${ }^{2}$ & $21.45 \pm 3.32$ \\
Gestational weight gain, kg & $15.78 \pm 5.06$ \\
GDM & $4,714(13.68)$ \\
PIH & $2,425(7.04)$ \\
Cesarean section & $14,117(40.95)$ \\
Gestational age, weeks & $38.82 \pm 1.35$ \\
Preterm & $1,458(4.23)$ \\
Neonatal birth weight, g & $3,379 \pm 447$ \\
Macrosomia & $2,494(7.24)$ \\
LGA & $6,668(19.34)$ \\
LBW & $906(2.63)$ \\
SGA & $732(2.12)$
\end{tabular}

Values are presented as mean $\pm \mathrm{SD}$ or $n(\%)$. BMI, body mass index; GDM, gestational diabetes mellitus; $\mathrm{PIH}$, pregnancyinduced hypertension; LGA, large for gestational age; LBW, low birth weight; SGA, small for gestational age.

The suggested GWG ranges according to the different methods are presented in Table 3. In method 1 , 16,058 women with optimal perinatal outcomes (46.59\%) were included in calculating the suggested GWG. The interquartile (25-75\%) range of GWG in each PPBMI group was proposed as the suggested GWG. The suggested GWGs calculated using this method overall were higher than the corresponding IOM-recommended GWGs, especially in the overweight and obese groups. Figure 2 shows the estimated isolated risk of SGA and LGA, as well as the total risk of SGA and LGA by GWG, according to method 2. Interestingly, in the underweight group, the curve for total risk of LGA and SGA against GWG appears to assume a U-shaped form. In all the other PPBMI groups, due to the much higher prevalence of LGA than of SGA, the curves for total risk of LGA and SGA were significantly skewed to the left, resulting in either no nadir or negative GWG being the nadir. The suggested GWG ranges determined by methods 3 and 4 were the same for the underweight group, while method 3 yielded a wider range for the other groups (Table 3; online suppl. Tables 1, 2; see www.karger.com/doi/10.1159/000509134 for all online suppl. material). Compared to the GWG according to the IOM guideline, the suggested GWG range according to methods 3 and 4 generally was wider and lower in all groups. 


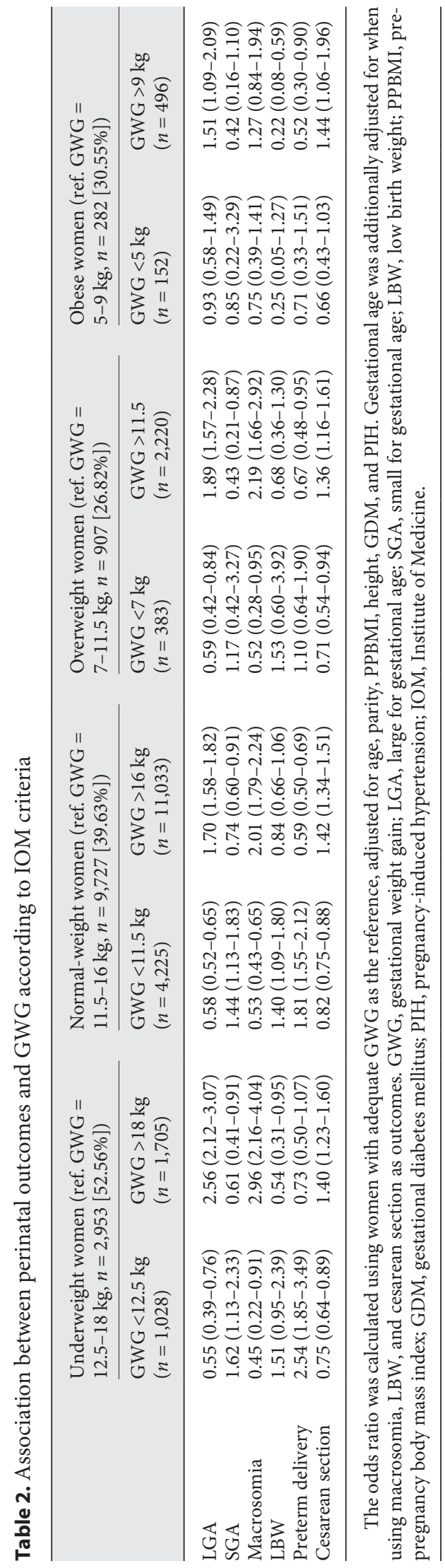

The percentages of women who met the IOM recommendation or the four suggested GWG ranges, as well as the corresponding prevalence rates for LGA and SGA, are presented in Table 4 and Figure 3. In summary, 38.4\% of the total number of participants met the IOM criteria. Approximately 51.73, 62.03, 65.74, and $39.75 \%$ of the participants met the suggested GWG ranges determined by methods $1-4$, respectively.

Method 1 resulted in more women within range compared to the IOM guideline in all PPBMI groups. There was no difference in risk of SGA between the groups. However, in the normal-weight and overweight groups, using method 1 led to a higher prevalence of LGA among women within the suggested GWG range than among those within the IOM-recommended range. Method 2 yielded far more women within the GWG range in the underweight and normal-weight groups, but far fewer women within the GWG range in the overweight group, when comparing the numbers of women to those based on the corresponding IOM-recommended range. No difference in SGA or LGA was observed when comparing method 2 and the IOM criteria. Using method 3, our study identified more women within range in all groups. Except that method 4 detected fewer cases of LGA in the normal-weight group compared to the IOM criteria, the prevalence rates of LGA and SGA as revealed by method 3 or 4 were generally similar to those revealed by the IOM criteria in all PPBMI groups.

\section{Discussion}

The current recommendation on GWG based on the IOM criteria appears to be less representative in Asian populations, as shown in recent studies in which only a small proportion of pregnant women were able to achieve GWG within the IOM-recommended ranges [10, 16]. The current study identifies "optimal" GWG using four distinct methods by weighing the risks of adverse pregnancy outcomes (LGA and SGA) in different PPBMI groups in a large cohort of Chinese women. We also compared the risks of LGA and SGA among women within optimal GWG as determined by different criteria.

A study by Kim et al. [17] discovered that among all modifiable risk factors, preventing excessive GWG has the greatest potential of reducing the incidence of LGA. Consistent with previous findings, our study found that GWG is positively associated with the risk of SGA, LBW, and preterm delivery, but negatively associated with the risk of LGA, macrosomia, and cesarean delivery. There- 


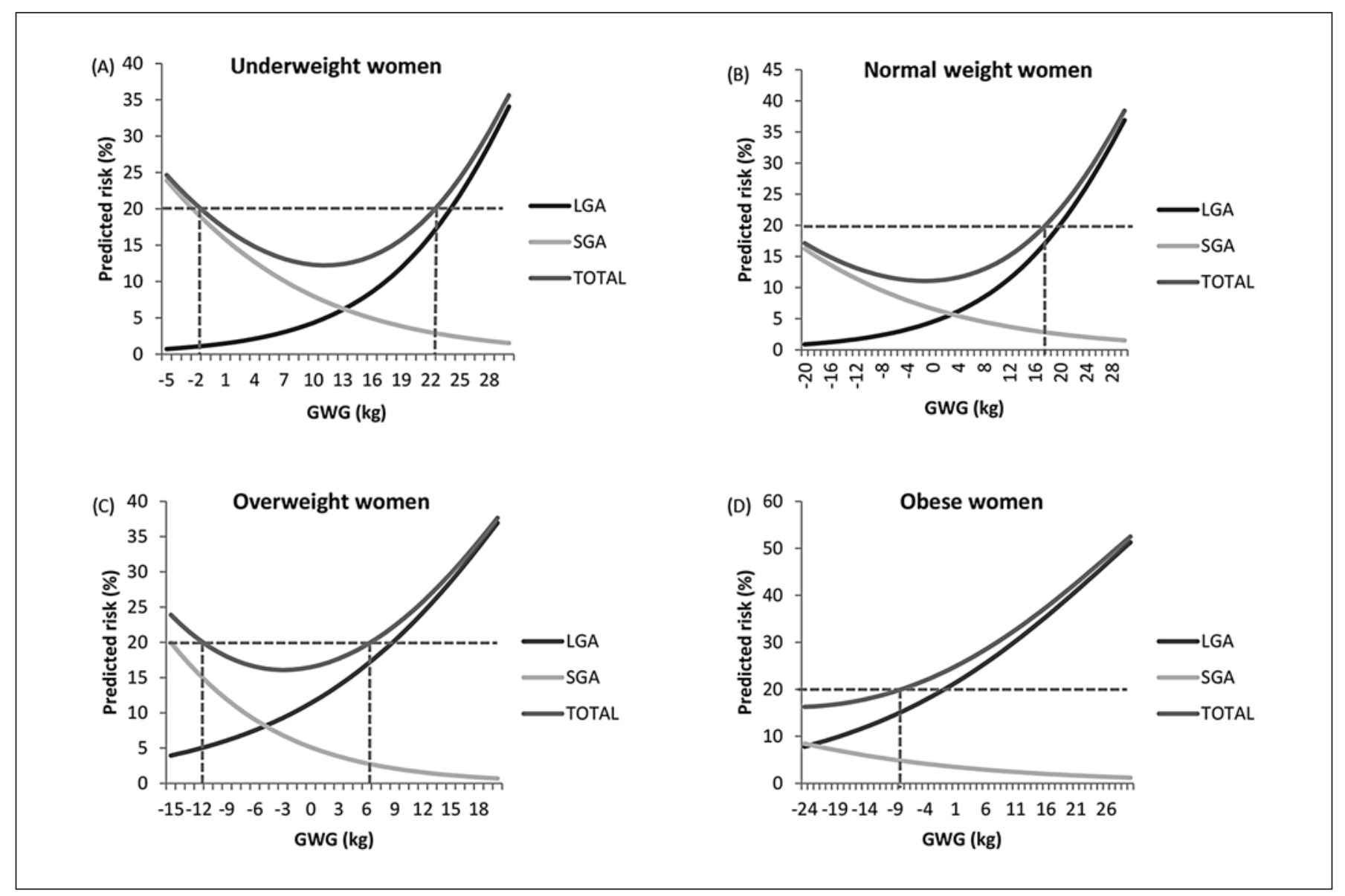

Fig. 2. A-D Predicted joint risk of LGA and SGA in the four prepregnancy body mass index groups by gestational weight gain. LGA, large for gestational age; SGA, small for gestational age; GWG, gestational weight gain.

Table 3. Suggested GWG range $(\mathrm{kg})$ by different strategies

\begin{tabular}{|c|c|c|c|c|c|c|}
\hline & Total, $n$ & IOM criteria & \multicolumn{4}{|c|}{ Suggested GWG by } \\
\hline $18.5 \mathrm{~kg} / \mathrm{m}^{2} \leq \mathrm{BMI}<25 \mathrm{~kg} / \mathrm{m}^{2}$ & 24,547 & $11.5-16$ & $12.5-18.5$ & to 17 & $9-19$ & $9-15$ \\
\hline $25 \mathrm{~kg} / \mathrm{m}^{2} \leq \mathrm{BMI}<30 \mathrm{~kg} / \mathrm{m}^{2}$ & 3,382 & $7-11.5$ & $9.5-16$ & to 6 & $4-12$ & $4-10$ \\
\hline $\mathrm{BMI} \geq 30 \mathrm{~kg} / \mathrm{m}^{2}$ & 923 & $5-9$ & $6.5-14$ & - & $0-12$ & $0-6$ \\
\hline
\end{tabular}

BMI, body mass index; GWG, gestational weight gain; IOM, Institute of Medicine; AGA, appropriate for gestational age; LBW, low birth weight; SGA, small for gestational age; LGA, large for gestational age; OR, odds ratio. ${ }^{\text {a }}$ Suggested GWG by method 1 refers to interquartile weight range among participants with adequate perinatal outcomes. Adequate perinatal outcomes indicate vaginal delivery of a term AGA infant without macrosomia or LBW. ${ }^{\text {b }}$ In method 2, logistic regression models were constructed to assess risk of SGA and LGA with respect to GWG (as a continuous variable), adjusted for age and parity. Suggested GWG was identified by a joint predicted risk of $20 \%$ for the occurrence of either SGA or LGA. ${ }^{c}$ In method 3, suggested GWG was identified by a significantly increased OR of 2.0 for the risk of LGA/SGA compared to the reference group. The reference group was defined according to IOM-recommended upper and lower limits of GWG (the ORs for LGA were calculated using $11.0-12.9 \mathrm{~kg}, 11.0-11.9 \mathrm{~kg}, 6.0-7.9 \mathrm{~kg}$, and $3.0-5.9 \mathrm{~kg}$ as the reference groups, while the ORs for SGA were calculated using $16.0-17.9 \mathrm{~kg}, 15.0-15.9 \mathrm{~kg}, 10.0-11.9 \mathrm{~kg}$, and $6.0-8.9 \mathrm{~kg}$ as the reference groups in the four BMI groups). ${ }^{\mathrm{d}}$ In method 4 , the same models as in method 3 were constructed, and a significantly increased OR for LGA $(\mathrm{OR}=1.5)$ and SGA $(\mathrm{OR}=2.0)$ compared to the reference group was defined to be the upper/lower range of suggested GWG. 


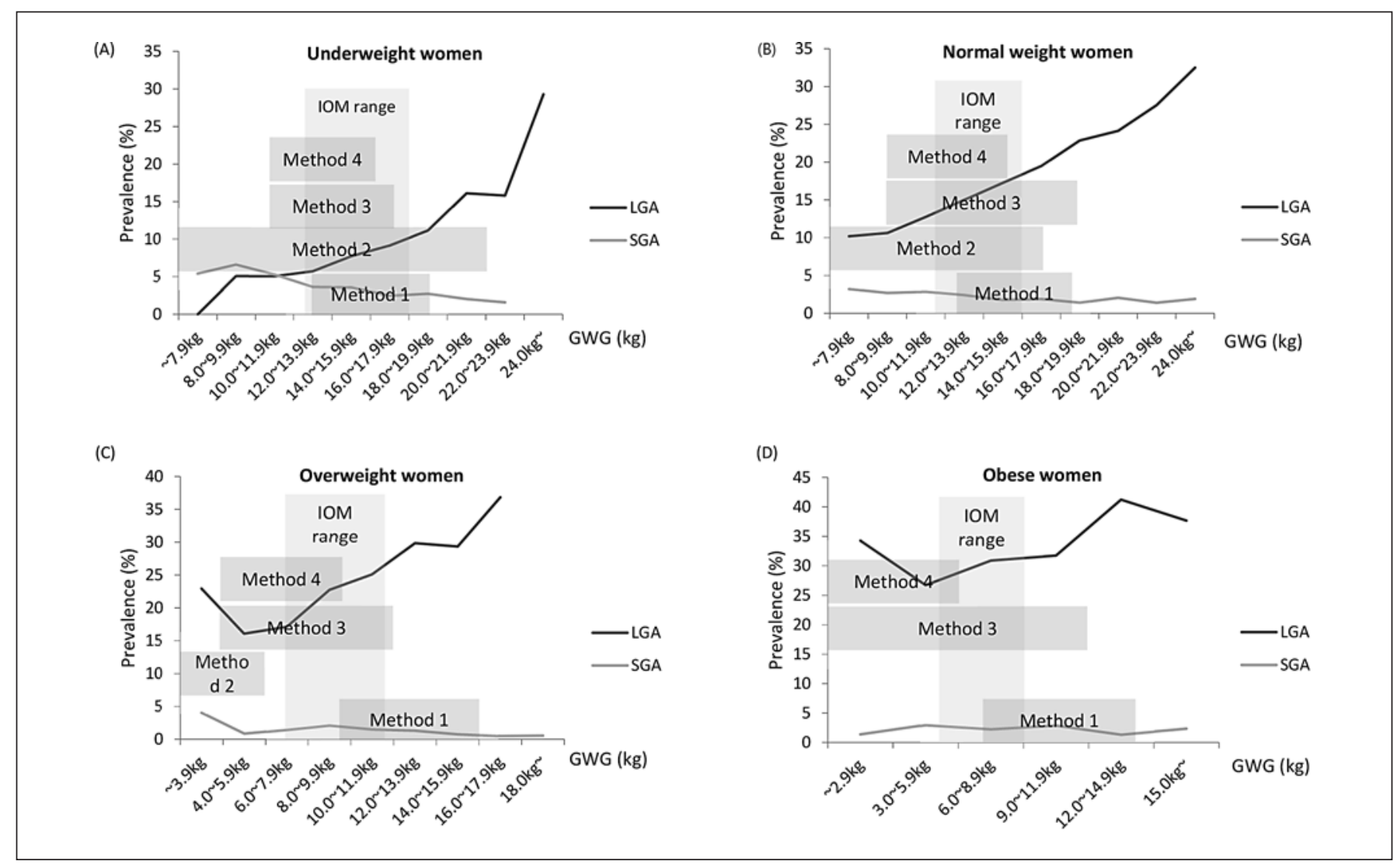

Fig. 3. A-D Four suggested GWG ranges and the corresponding prevalence rates of LGA and SGA. LGA, large for gestational age; SGA, small for gestational age; GWG, gestational weight gain.

fore, determining optimal GWG is mainly to balance the risks of these contrasting pregnancy outcomes, especially LGA and SGA in the current study.

In this study, the different analytical methods resulted in distinct suggested GWG ranges. Among mothers who have optimal pregnancy outcomes, the interquartile method is a common strategy for calculating "optimal" GWG [18]. Indeed, the IOM Committee used this strategy to calculate "optimal" GWG for twin pregnancies [7]. The suggested GWG according to this method was substantially higher than the IOM-recommended ranges, especially in the overweight and obese groups, which was similar to the findings by Wang et al. [19]. A possible explanation is that we did not exclude women with longterm weight retention or offspring obesity due to lack of related information $[20,21]$. Possibly owing to the high "optimal" GWG determined by the interquartile method, the prevalence of LGA was higher among women who met the suggested GWG by this method compared to those who met the IOM criteria.
Predicted joint risk of $20 \%$ for SGA and LGA delivery is another generally used strategy for calculating "optimal" GWG $[15,22,23]$. However, due to the high prevalence of LGA and the low prevalence of SGA in this study, the linear fitting model did not identify clinically applicable cut-off values for the lower GWG limit in all weight groups. Therefore, it appears that method 2, which uses GWG as a continuous variable and a $20 \%$ risk tolerance, may not be applicable to this population. Consequently, we developed models using GWG as a categorized variable (methods 3 and 4). The suggested GWG range according to method 3 appears to cover the largest proportion of participants of all the four methods. In all, $62.03 \%$ of the participating subjects were considered to have appropriate GWG by method 3, which was higher than the proportion determined by IOM criteria $(51.73 \%)$, but without a significantly increased risk of LGA or SGA. A high prevalence of LGA and macrosomia was observed in this study, which was similar to a national survey result for 101,723 neonates [24]. LGA is associated with both 


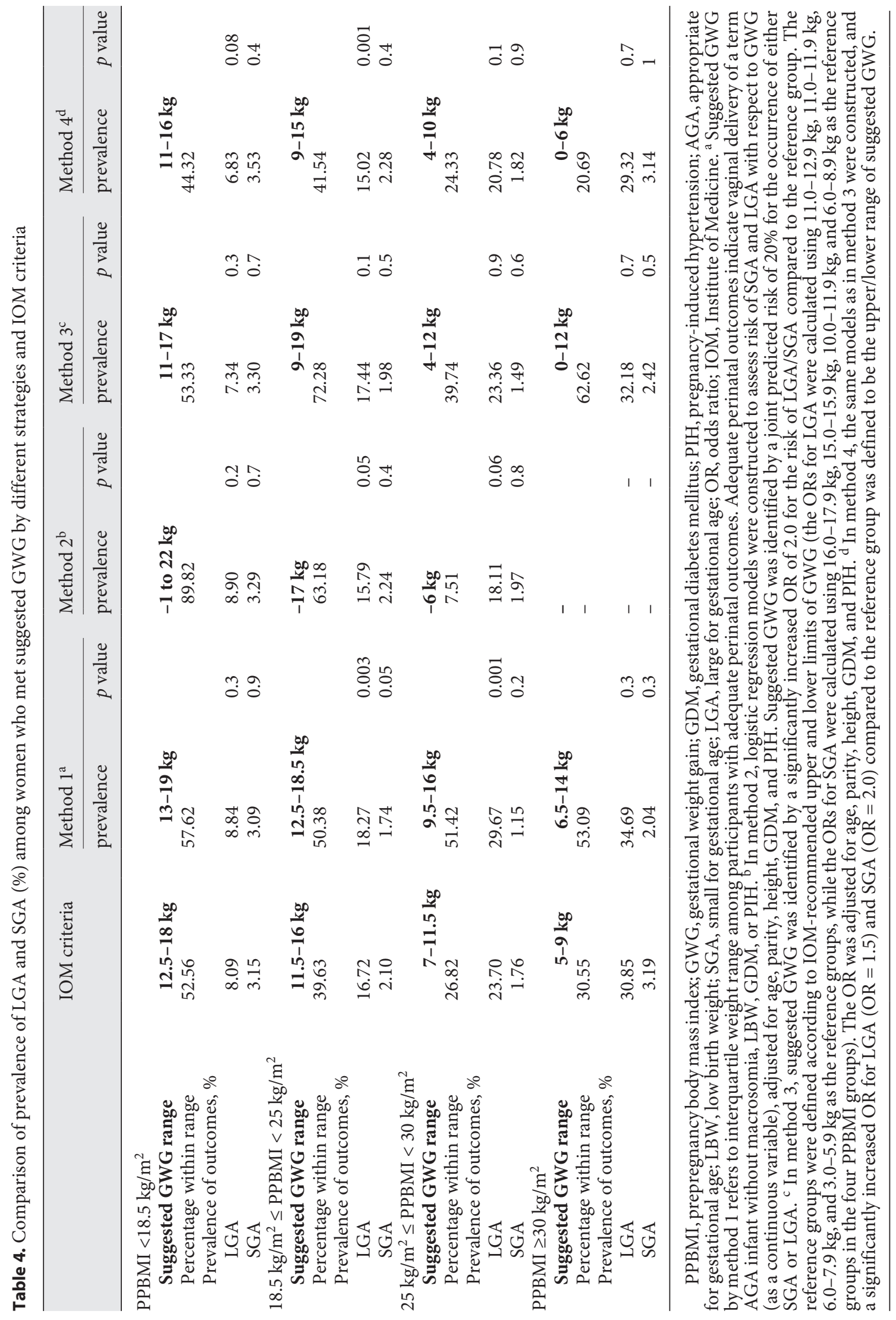


perinatal complications and long-term risks of obesity and metabolic diseases of offspring later in life [25]. We thus placed a relatively higher value on reducing LGA prevalence than on reducing SGA prevalence in our study population by applying method 4 .

We used four different methods to calculate suggested GWG and made comparisons to IOM-recommended GWG. Recent evidence has suggested that IOM GWG targets may be overly generous for obese Asian women. A study of Singaporean women suggested a total GWG of $\leq 1.8 \mathrm{~kg}$ for obese women [9]. A Japanese guideline suggested a GWG of 5-7 kg for women with a PPBMI $>24.0$ $\mathrm{kg} / \mathrm{m}^{2}$ [26]. Similarly, a systematic review by Faucher and Barger [27] recommended a GWG of $1-5 \mathrm{~kg}$ for class II obesity and no GWG for class III obesity. Therefore, a lower GWG than recommended by the IOM guideline may be appropriate for overweight and obese Chinese women. Additionally, the high birth weight according to the national survey [24], as well as the high LGA prevalence even in the low-GWG group in this study, suggest that overweight and obese Chinese women should probably be allowed to gain less weight to limit the risk of LGA. Conversely, another systematic review reported that even though GWG below the IOM guideline in obese women was accompanied by decreased odds of LGA, it was also associated with increased odds of SGA and preterm delivery [28]. We did not observe any increased risk of SGA when applying the suggested GWG ranges according to methods 3 and 4 to the overweight and obese groups. However, the statistical power to determine risk of SGA may have been limited for overweight and obese women.

There is a lack of data on recommended GWG in Chinese women. Tsai et al. [8] suggested a GWG of 10-14 kg for women with an appropriate PPBMI in Taiwan, which is similar to the results from method 4 in this study. Wang et al. [19] proposed a modest GWG (10-15 kg) in normalweight women, but a higher GWG for overweight (8-14 $\mathrm{kg}$ ) and obese Chinese women (5-11.5 kg), compared to the IOM guideline using $\mathrm{P}_{25}-\mathrm{P}_{75}$ of GWG for women in China. However, the proposed GWGs for overweight and obese women may not be accurate due to the limited numbers of participants. Additionally, recall bias is a concern due to the cross-sectional study design.

Our study has certain limitations. First, no long-term risks of obesity and cardiometabolic health parameters were evaluated in the mothers and offspring in our study, due to lack of information. Second, our study was completed in Northern China and may not be representative of a broader Chinese population. Third, the sample size of the overweight and obese groups is rather small in this study.
In conclusion, this study suggests a modest GWG goal compared to IOM recommendations based on a large, prospective cohort of Chinese women. Conventional methods may be unsuitable for calculating "optimal" GWG in a population with notably high LGA prevalence rates. Our suggested GWG will need to be confirmed in a larger and more diverse population.

\section{Acknowledgements}

We thank the participants for their participation and the medical staff for their work on information collection.

\section{Statement of Ethics}

The study was conducted ethically in accordance with the World Medical Association Declaration of Helsinki. The study protocol was approved by the Ethics Committee of Beijing Obstetrics and Gynecology Hospital (2018-KY-009-01) and written informed consent was obtained from every participant.

\section{Conflict of Interest Statement}

The authors declare no conflict of interest.

\section{Funding Sources}

This study was funded by the National Key Research and Development Program (2016YFC1000304), the National Natural Science Foundation of China (grant No. 81671477), and the Capital's Funds for Health Improvement and Research (CFH 2018-2-2112).

\section{Author Contributions}

W. Zheng conducted the population study, analyzed and interpreted the data, and drafted the manuscript. W.H. participated in data analysis and interpretation, and draft revision. L.Z. and Z.T. participated in data collection and interpretation of the results. Q.Y. and T.W. assisted with data collection and analysis. G.L. and W. Zhang designed the study and critically revised the manuscript. All authors read and approved the final manuscript.

\section{Data Availability Statement}

The data set used in this study is accessible upon reasonable request to the corresponding author via liguanghui@ccmu.edu.cn. 


\section{References}

1 Voerman E, Santos S, Inskip H, Amiano P, Barros H, Charles MA, et al.; LifeCycle Project-Maternal Obesity and Childhood Outcomes Study Group. Association of Gestational Weight Gain with Adverse Maternal and Infant Outcomes. JAMA. 2019 May; 321(17):1702-15.

2 Widen EM, Whyatt RM, Hoepner LA, Ramirez-Carvey J, Oberfield SE, Hassoun A, et al. Excessive gestational weight gain is associated with long-term body fat and weight retention at 7 y postpartum in African American and Dominican mothers with underweight, normal, and overweight prepregnancy BMI. Am J Clin Nutr. 2015 Dec;102(6): 1460-7.

3 Goldstein RF, Abell SK, Ranasinha S, Misso M, Boyle JA, Black MH, et al. Association of Gestational Weight Gain with Maternal and Infant Outcomes: A Systematic Review and Meta-Analysis. JAMA. 2017 Jun;317(21): 2207-25.

4 Rong K, Yu K, Han X, Szeto IM, Qin X, Wang $J$, et al. Pre-pregnancy BMI, gestational weight gain and postpartum weight retention: a meta-analysis of observational studies. Public Health Nutr. 2015 Aug;18(12):2172-82.

5 Oken E, Taveras EM, Kleinman KP, Rich-Edwards JW, Gillman MW. Gestational weight gain and child adiposity at age 3 years. Am J Obstet Gynecol. 2007 Apr;196(4):322.e1-8.

6 Scott C, Andersen CT, Valdez N, Mardones F, Nohr EA, Poston L, et al. No global consensus: a cross-sectional survey of maternal weight policies. BMC Pregnancy Childbirth. 2014 May;14(1):167.

7 Rasmussen KM, Yaktine AK, Rasmussen KM, Yaktine AK. Weight gain during pregnancy: reexamining the guidelines. National Academies Press; 2009.

8 Tsai IH, Chen CP, Sun FJ, Wu CH, Yeh SL. Associations of the pre-pregnancy body mass index and gestational weight gain with pregnancy outcomes in Taiwanese women. Asia Pac J Clin Nutr. 2012;21(1):82-7.

9 Ee TX, Allen JC Jr, Malhotra R, Koh H, Østbye T, Tan TC. Determining optimal gestational weight gain in a multiethnic Asian population. J Obstet Gynaecol Res. 2014 Apr;40(4): 1002-8.
10 Morisaki N, Nagata C, Jwa SC, Sago H, Saito $\mathrm{S}$, Oken E, et al. Pre-pregnancy BMI-specific optimal gestational weight gain for women in Japan. J Epidemiol. 2017 Oct;27(10):492-8.

11 National Diabetes Data Group. Classification and diagnosis of diabetes mellitus and other categories of glucose intolerance. Diabetes. 1979 Dec;28(12):1039-57.

12 Metzger BE, Gabbe SG, Persson B, Buchanan TA, Catalano PA, Damm P, et al.; International Association of Diabetes and Pregnancy Study Groups Consensus Panel. International Association of Diabetes and Pregnancy Study Groups recommendations on the diagnosis and classification of hyperglycemia in pregnancy. Diabetes Care. 2010 Mar;33(3):67682.

13 Arulkumaran N, Lightstone L. Severe pre-eclampsia and hypertensive crises. Best Pract Res Clin Obstet Gynaecol. 2013 Dec;27(6): 877-84.

14 Villar J, Cheikh Ismail L, Victora CG, Ohuma EO, Bertino E, Altman DG, et al.; International Fetal and Newborn Growth Consortium for the 21st Century (INTERGROWTH-21st). International standards for newborn weight, length, and head circumference by gestational age and sex: the Newborn Cross-Sectional Study of the INTERGROWTH-21st Project. Lancet. 2014 Sep;384(9946):857-68.

15 Beyerlein A, Schiessl B, Lack N, von Kries R. Optimal gestational weight gain ranges for the avoidance of adverse birth weight outcomes: a novel approach. Am J Clin Nutr. 2009 Dec; 90(6):1552-8

16 Li C, Liu Y, Zhang W. Joint and Independent Associations of Gestational Weight Gain and Pre-Pregnancy Body Mass Index with Outcomes of Pregnancy in Chinese Women: A Retrospective Cohort Study. PLoS One. 2015 Aug;10(8):e0136850.

17 Kim SY, Sharma AJ, Sappenfield W, Wilson HG, Salihu HM. Association of maternal body mass index, excessive weight gain, and gestational diabetes mellitus with large-forgestational-age births. Obstet Gynecol. 2014 Apr;123(4):737-44.

18 Wong W, Tang NL, Lau TK, Wong TW. A new recommendation for maternal weight gain in Chinese women. J Am Diet Assoc. 2000 Jul;100(7):791-6.

19 Wang J, Duan Y, Pang X, Jiang S, Yin S, Yang $\mathrm{Z}$, et al. Gestational weight gain and optimal ranges in Chinese mothers giving singleton and full-term births in 2013. Chin J Prev Med. 2018 Jan;52(1):31-7.
20 Nehring I, Schmoll S, Beyerlein A, Hauner H, von Kries R. Gestational weight gain and long-term postpartum weight retention: a meta-analysis. Am J Clin Nutr. 2011 Nov; 94(5):1225-31.

21 Sridhar SB, Darbinian J, Ehrlich SF, Markman MA, Gunderson EP, Ferrara A, et al. Maternal gestational weight gain and offspring risk for childhood overweight or obesity. Am J Obstet Gynecol. 2014 Sep;211(3):259.e1-8.

22 Choi SK, Lee G, Kim YH, Park IY, Ko HS, Shin JC. Determining optimal gestational weight gain in the Korean population: a retrospective cohort study. Reprod Biol Endocrinol. 2017 Aug;15(1):67.

23 Hirooka-Nakama J, Enomoto K, Sakamaki K, Kurasawa K, Miyagi E, Aoki S. Optimal weight gain in obese and overweight pregnant Japanese women. Endocr J. 2018 May;65(5): 557-67.

24 Li G, Kong L, Li Z, Zhang L, Fan L, Zou L, et al. Prevalence of macrosomia and its risk factors in china: a multicentre survey based on birth data involving 101,723 singleton term infants. Paediatr Perinat Epidemiol. 2014 Jul; 28(4):345-50.

25 Skilton MR, Siitonen N, Würtz P, Viikari JS, Juonala M, Seppälä I, et al. High birth weight is associated with obesity and increased carotid wall thickness in young adults: the Cardiovascular Risk in Young Finns study. Arterioscler Thromb Vasc Biol. 2014 May;34(5): 1064-8.

26 Tsukamoto H, Fukuoka H, Inoue K, Koyasu M, Nagai Y, Takimoto H. Restricting weight gain during pregnancy in Japan: a controversial factor in reducing perinatal complications. Eur J Obstet Gynecol Reprod Biol. 2007 Jul;133(1):53-9.

27 Faucher MA, Barger MK. Gestational weight gain in obese women by class of obesity and select maternal/newborn outcomes: a systematic review. Women Birth. 2015 Sep; 28(3):e70-9.

28 Kapadia MZ, Park CK, Beyene J, Giglia L, Maxwell C, McDonald SD. Can we safely recommend gestational weight gain below the 2009 guidelines in obese women? A systematic review and meta-analysis. Obes Rev. 2015 Mar;16(3):189-206. 